\title{
International Summer School Session on the Atmospheric Boundary Layer Les Houches, France, 17-27 June 2008
}

In this summer school, PhD students and young scientists will be introduced to the latest developments in theoretical concepts, observations and numerical techniques in boundary-layer meteorology. Outstanding lecturers will present key concepts and issues and, in the modelling and simulation session, participants will receive hands-on experience with a selection of numerical techniques to study boundary-layer flows.

The school is limited to a maximum of 50 students. A tuition fee of $€ 600$ is due, which also covers accommodation, meals and study material.

If you are interested in this course, please send an email to h.vandop@phys.uu.nl and we will identify you as a potential participant.

For more information and registration we refer to the website http://www.phys.uu.nl/^dop/ summerschool/

Han van Dop, Institute for Marine and Atmospheric research Utrecht (IMAU), Utrecht University, The Netherlands Bert Holtslag, Meteorology and Air Quality Section, Wageningen University, The Netherlands Jordi Vilà, Meteorology and Air Quality Section, Wageningen University, The Netherlands 